\title{
THE SUSPENSION SYSTEM WITH PRESSURE CONTROL USED FOR LOWER LIMB PROSTHESES
}

\author{
Spanu Alina Rodica ${ }^{1}$, Besnea Daniel ${ }^{2}$, Moraru Edgar ${ }^{3}$ \\ 1,2,3 University Politehnica of Bucharest, Faculty of Mechatronics and Mechanical Engineering, \\ Mechatronics and Precision Engineering Department \\ Spanualina@yahoo.com, d bes@yahoo.com,Eddymilan91@yahoo.com
}

\begin{abstract}
The paper aims to provide a new genuine solution for the modular personalized prostheses for the lower limb, whose suspension system will be based on the pneumotronic system required to controll the pressure forces acting on the injured lower limb. The mechatronic system involves the sensor system too, so that there are used the force sensor types. Due to these reasons, an intermediate socket will be used on which will be put a network of pellet components supplied with controlled values of pressure. The new and genuine technical aspects will be: the pressure force control for the suspension system; the necessity of adjusting the prostheses to the shape of injured lower limb with variable volum, personalized for each person.
\end{abstract}

Keywords: Prosthesis, Lower Limb, Mechatronic System, Pressure Control.

\section{Introduction}

Nowadays many people experienced the lower limb missing due to some causes such injuries, wars, fights or congenital issues for instance. The posttraumatic therapy is hardly and involves some supplementary apparatus and devices, the presence of qualified personnel and much time too. The transtibial prosthesis is essential for the patient comfort, so that it should be designed accurately in order to satisfy many requirements. Finally, the prosthesis could be considered like a feed-back mechatronic system involving some sensors, electric or pneumatic motors, controllers as well as specific types of materials.

The paper [7] describes the study made in order to evaluate the multi-material 3D model of transtibial residuum for the patient analysing the loading conditions on the residuum from a prosthetic socket. It was made a $3 \mathrm{D}$ model for the material with specific parameters and the FEA analysis study was conducted for the surface and internal geometry. The magnetic resonance imaging (MRI) was used for data acquisitions with markers placed on the skin along the residual limb.

The prosthesis socket is the most important component of this mechatronic system, so the suspension accuracy and the way of fitting in the residual limb should be controlled. The variable volume of the residual limb, should be another reason for controlling the pressure acting on the patient skin, otherwise some risks of trauma may occur. The paper [1] presents the portable instrument developed based on bioimpedance system to acquire the fluid volume changes of the residual limb outside the lab, during walking.

In order to analyse the variation of pressure forces inside the socket some studies describe the sensor system used by the researchers. The paper [6] described the results obtained after the evaluation of three types of force sensitive resistors (FSR) available in thin film polymer for biomechanical measurement.

The sensor system used in paper [2] has the main components the interface sensor system and data acquisition unit (DAQ) consisting of digital signal processors, sensor system controller, Bluetooth Radio and the personal computer with the software. A clinical test was conducted by collecting real time data for the pressure acting on the lower limb residuum.

The paper [8] presents a strategy for instrumenting three sensor types placed inside the digitized socket. There were used proximity sensors to identify the limb presence, force sensing resistors for quantifying the pressure and inductive sensors to measure the limb-to-socket distances.

The paper [3] presents the way the patient could adjust the distance between the limb and the socket by using a cable to move socket panels radially inwards toward the residual limb. The paper [5] proposed the wireless technique using the mobile phone in order to adjust the socket distance.

Even though the distance inside the socket and the pressure forces are controlled, we have to pay attention to the suspension system of the prostheses. This system, whose important role is to attach the prosthetic limb to the body, has to prevent excessive translations, rotations and vertical moments 
between the residual limb and the socket. Although a number of prosthetics suspension system are available, the selection criteria is based mainly on subjective experience.

The paper [9] described the vacuum suspension system using the vacuum pump actuated by the gravity force of the patient. The vacuum suspension is the most used for the lower residual limb prosthesis.

The paper [4] described the magnetic prosthesis suspension system that involves electromagnets embedded in the socket with a feed-back loop implemented in order to control the force between the electromagnet and the magnetic material embedded in the liner.

A pressure sensor and a microcontroller has to adjust the force acting on the patient skin.

The results show that the electromagnetic suspension is adequate to maintain the contact between the liner and the socket.

The paper [7 contract] made a bibliographic review regarding the suspension types used for prosthetics. Finally, the qualified personnel decided suspension type according to the shape of residual limb, cylinder or cone, the muscle strength, the skin soft, some external rigid shapes existing on the residual limb, the requirement of activities with high mobility level.

This paper aims to describe a new, genuine solution for the suspension system controlling the pressure force acting on the patient residual limb skin.

The main advantages are regarding the variable pressure force having the role of avoiding the variable fluid volume, meaning the distance limbsocket too, as well as the possibility of improving the circulatory sanguine system of the patient.

\section{The Proposed Solution for the Suspension System}

The proposed solution refers to a mechatronic system for the residual limb suspension, comprising a network of pellet placed inside the intermediate socket of the lower limb prosthesis.

The pressure inside the pellet will be controlled by a pneumotronic system.

This solution has the advantage of maintaining the constant pressure force acting on the patient skin, avoiding the injuries or some other troubles affecting the comfort. Meantime, due to the control system the socket is centred relative to the external hard socket of the prostheses.

The most important consequence is that there are not external forces or couples, which could influence the patient mobility.

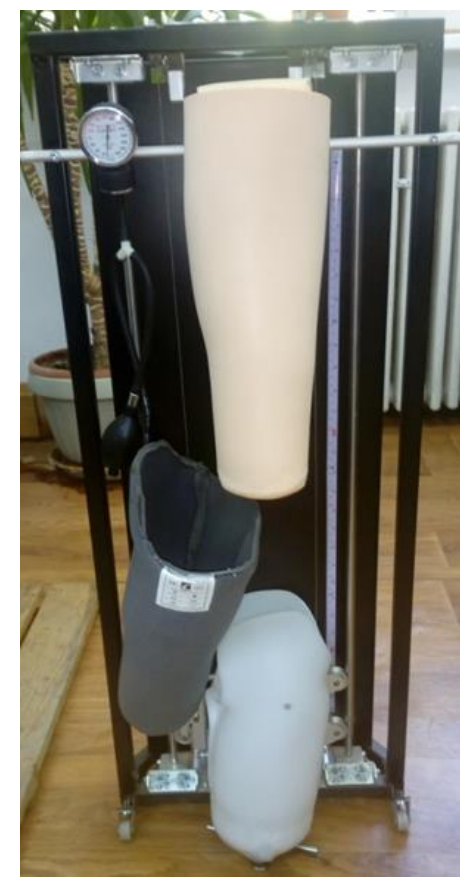

Figure 1: The grey intermediate socket and the white hard socket of the prosthesis.

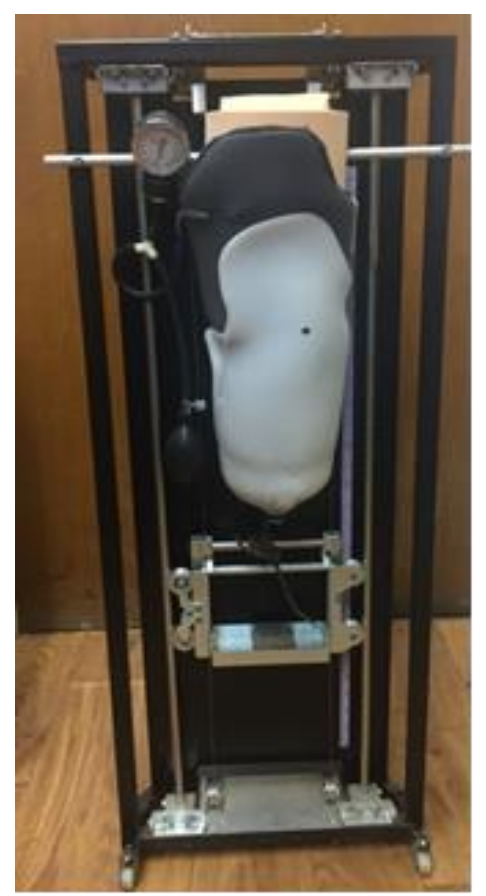

Figure 2: The sockets are assembled on the residual limb.

Another advantage of this solution is the possibility of personalized shape of contact point positions along the residual limb, when the skin has some other injuries, making an external massage.

As we may infer from the Fig. 1 the components of the prosthesis are: the grey socket with the network of pellets inside it and the white hard socket that will be in contact with the prosthesis leg. 
In the Fig. 2, the socket with the network is placed on the residual limb, so due to the pressure forces and friction forces of the elastic material, we have maintained a permanent contact with the residual limb.

\section{Experimental Setup}

For the experimental setup we have designed the mechatronic system comprising the pressure source with the pressure controller, whose role is to maintain a constant value of air pressure inside the pellet. The position of inflated pellets on the internal surface of the socket could be controlled too. In order to measure the pressure force we have used the resistance force sensor and the Arduino microcontroller.

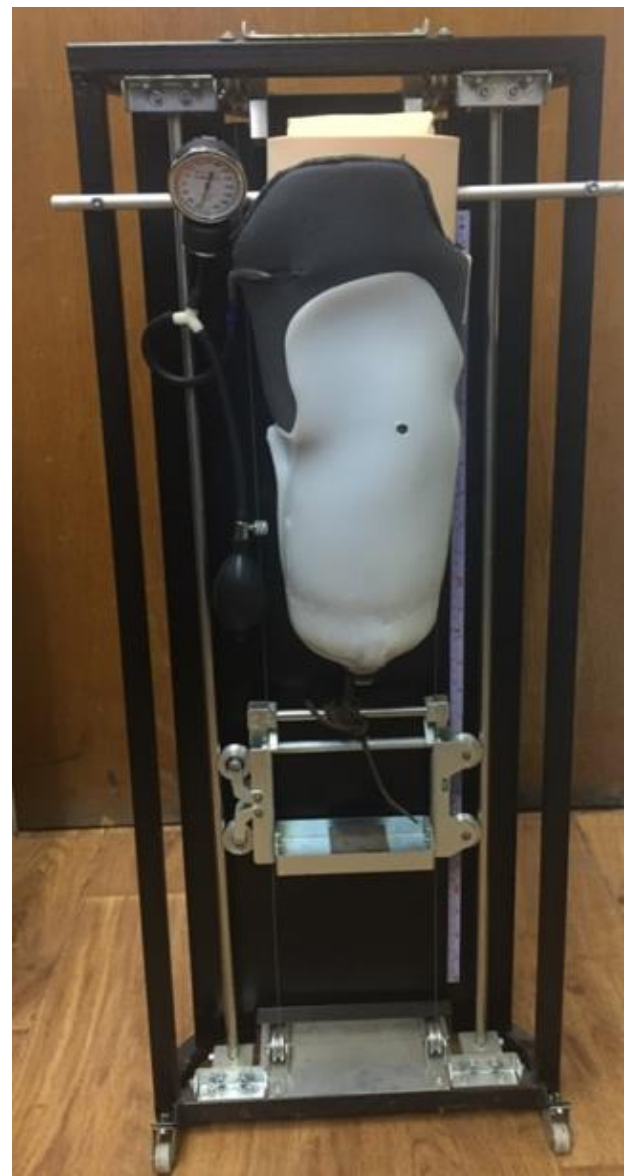

Figure 3: The tested prostheses.

As it is shown in the Fig. 3, we put the prosthesis to the test of weight that could be supported without losing the contact with the residual limb.

The Fig. 4 presents the variation of the weight put on the hard white socket until the contact with the residual limb will be lost.

The experiments showed that the maximum value was $\mathrm{F}=1.8[\mathrm{~N}]$, which is acceptable for prosthesis functional parameters.

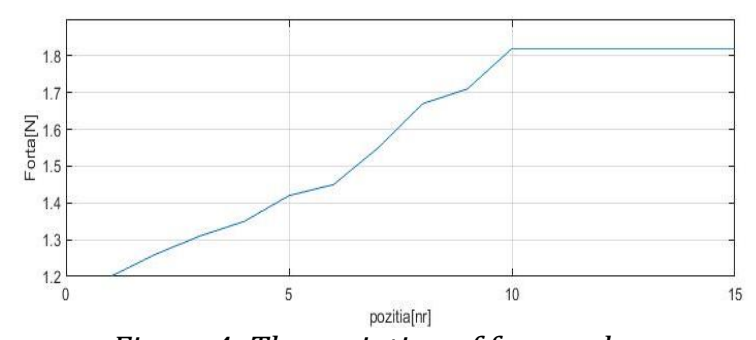

Figure 4: The variation of force values

The next stage of this research was about measuring the variation of pressure force acting on the contact points with the residual limb. At first we have used the Arduino microcontroller and the force sensor SEN-09375 ROHS (Fig. 5) for calibration.

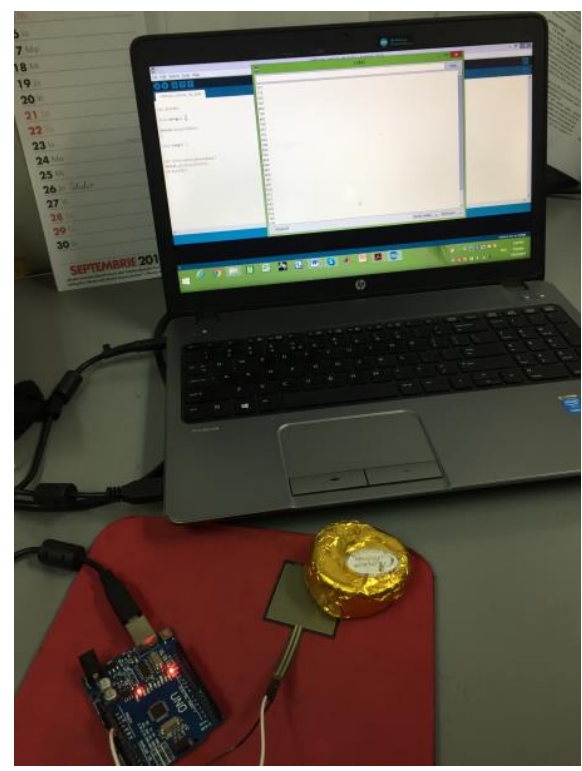

Figure 5: The sensor calibration

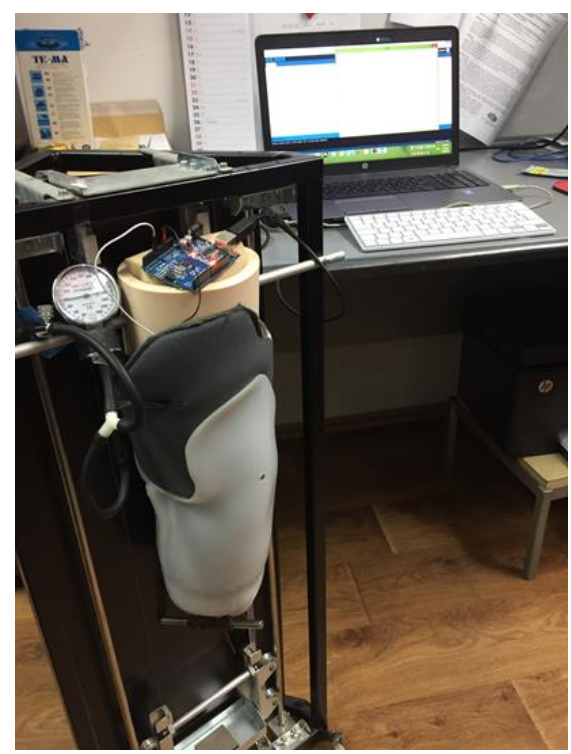

Figure 6: The experimental setup for measuring the pressure force 
Finally, we have measured the pressure force values inside the grey socket, when the pellets are in contact with the residual limb by using the microcontroller and the force sensor (Fig. 6). The maximum pressure force developed on one pellet, acting on the patient skin was about $0.031 \mathrm{~N}$, which is an acceptable value affording the contact with the residual limb.

\section{Conclusions}

The paper aims to point out the solution for pneumatic suspension used for lower limb prostheses. The main advantages refer to: the pressure force control for maintaining the contact with the residual limb; the control of inflated pellet position around the working surface of the socket; the external massage of the skin; the avoidance of fluid volume of the residual limb during walking.

There are not supplementary forces or couples acting on the residual limb, so we may conclude this solution could improve the patient comfort and mobility.

\section{References}

[1] Hinrichs, P., Cagle, J., C., Sanders, E., A portable bioimpedance instrument for monitoring residual limb fluid volume in people with transtibial loss: A technical note, Medical Engineering and Physics, 68, (2009), 101-107.

[2] Laszczak, P., Grath, M., Tang, J., Gao, J., Jiang, L., Bader, D., L., A pressure and shear sensor system for stress measurement at lower limb residuum/socket interface, Medical Engineering and Physics, 38, (2016), 695-700.
[3] McLean, J., Redd, Ch., Larsen, B., Garbini, J., Brzostowski, J., Hafner, B., Sanders, J., Socket size adjustments in people with transtibial amputation: effects on residual limb fluid volume and limb socket distance, Clinical Biomechanics, 63, (2019), 161-171.

[4] Ng, J., Johnson, J., Miller, S., Newton, K., Roe, T., Woo, R., Electromagnetic suspension system for control of limb volume in prosthetics, Procedia CIRP 65(2017) 180-183.

[5] Sanders, J., Garbini, J., McLean, J., Hinrichs, P., Predmore, T., Brzostowski, J., Redd, J., Cagle, J., $A$ motor-driven adjustable prosthetic socket operated using a mobile phone app: A technical note, Medical Engineering and Physics, 68, (2019), 94-100.

[6] Schofield, J., Evans, K., Hebert, J., Marasco, P., Carey, J., The effect of biomechanical variables on force sensitive resistor error: implications for calibration and improved accuracy, Journal of Biomechanics, 49, (2016), 786-792.

[7] Sengeh D., Moerman, K., Petron, A., Herr, H., Multi-material 3-D viscoelastic model for transtibial residuum from in-vivo indentation and MRI data, Journal of the Mechanical Behavior of Biomedical Materials, 59, (2016),379-392, accessed at:02.09.2019

[8] Swanson, E., McLean, J., Allyn, K., Redd, C., Sanders, J., Instrumented socket inserts for sensing interaction at the limb-socket interface, Medical Engineering and Physics, 51, (2018), 111-118,

[9] https://www.ottobock.ro/proteze/extremitate a-inferioar\%C4\%83/prezentaregeneral\%C4\%83/sistemul-de-vid-harmonypentru-protezele-transtibiale/, accessed at: 10.10.2019 